\title{
On soluble groups of automorphisms of nonorientable Klein surfaces
}

by

\author{
Grzegorz G romadzki (Bydgoszcz)
}

\begin{abstract}
We classify up to topological type nonorientable bordered Klein surfaces with maximal symmetry and soluble automorphism group provided its solubility degree does not exceed 4. Using this classification we show that a soluble group of automorphisms of a nonorientable Riemann surface of algebraic genus $\mathbf{q} \geq 2$ has at most $24(\mathbf{q}-1)$ elements and that this bound is sharp for infinitely many values of $\mathbf{q}$.
\end{abstract}

1. Introduction. By a Klein surface we mean in this paper a compact nonorientable surface $X$ equipped with a dianalytic structure, and by an automorphism a conformal or anticonformal homeomorphism [1]. The algebraic genus $\mathbf{q}$ of a Klein surface $X$ is defined as the genus of its canonical double Riemann cover [1] and it turns out that for a Klein surface of topological genus $g$, with $k(k \geq 0)$ boundary components, $\mathbf{q}=g+k-1$. It is well known [12] that a group of automorphisms of a compact Klein surface $X$ of algebraic genus $\mathbf{q} \geq 2$ has at most $84(\mathbf{q}-1)$ elements. Although this bound is known to be attained for infinitely many values of $\mathbf{q}$, none of the corresponding groups can be soluble and none of the corresponding surfaces can have boundary. If $X$ has nonempty boundary then $|G| \leq 12(\mathbf{q}-1)$. If $G$ is soluble then $|G| \leq 48(\mathbf{q}-1)$. This case for Riemann surfaces was studied in [6], [7] (see also [8]). Here we study soluble groups of automorphisms of nonorientable Riemann surfaces (i.e. nonorientable Klein surfaces without boundary). We show that the bound in this case is $24(\mathbf{q}-1)$. We also find an infinite series of $\mathbf{q}$ for which this bound is attained. Looking for the values of $\mathbf{q}$ for which this bound is attained we discovered a somewhat surprising fact: a soluble group of order $24(\mathbf{q}-1)$ acts as a group of auto-

1991 Mathematics Subject Classification: Primary 20F05, 20F16; Secondary 30F50.

Key words and phrases: Riemann surfaces, Klein surfaces, automorphism groups, soluble groups.

Paper written during the author's stay at the Complutense University of Madrid. 
morphisms on a nonorientable Riemann surface of algebraic genus $\mathbf{q}$ if and only if it can be viewed as an $M^{*}$-group acting on a nonorientable bordered Klein surface with maximal symmetry of algebraic genus $\mathbf{q}^{\prime}=2 \mathbf{q}-1$, having $k=\mathbf{q}-1$ boundary components (see Section 3 for definitions), which prompted the study those surfaces and groups. We classify topological types of nonorientable Klein surfaces with maximal symmetry and with soluble automorphism group provided its solubility degree does not exceed 4 . Notice that a group of automorphisms of a nonorientable Riemann surface can be viewed as a group of conformal automorphisms of an orientable Riemann surface of the same algebraic genus. Thus the bound $48(\mathbf{q}-1)$ for the order of a soluble group of automorphisms of an orientable Riemann surface is not attained in the nonorientable case. It is worth noting that this is in marked contrast with the corresponding results for the maximal groups of automorphisms of compact Klein surfaces for which absolute bounds $84(\mathbf{q}-1)$ in case of surfaces without boundary and $12(\mathbf{q}-1)$ in case of bordered surfaces are attained in both the orientable and nonorientable cases (see [20] and [22] respectively).

2. Preliminaries. We shall prove the announced results by means of NEC-groups. An $N E C$-group is a discrete subgroup $\Lambda$ of the group $\mathfrak{G}$ of isometries of the non-Euclidean plane $\mathbb{C}^{+}$(including those which reverse orientation: reflections and glide reflections) with compact quotient space $\mathbb{C}^{+} / \Lambda$. Let $\mathfrak{G}^{+}$denote the subgroup of index 2 in $\mathfrak{G}$ consisting of orientation preserving isometries. An NEC-group $\Lambda$ contained in $\mathfrak{G}^{+}$is called a Fuchsian group, and a proper $N E C$-group in the other case. In what follows $\Lambda^{+}=$ $\Lambda \cap \mathfrak{G}^{+}$is the canonical Fuchsian subgroup of an NEC-group $\Lambda$. NEC-groups were first studied by Wilkie [23] who associated with every such group a signature, which is a sequence $\tau$ of numbers and symbols of the form

$$
\tau=\left(g ; \pm ;\left[m_{1}, \ldots, m_{r}\right] ;\left\{\left(n_{11}, \ldots, n_{1 s_{1}}\right), \ldots,\left(n_{k 1}, \ldots, n_{k s_{k}}\right)\right\}\right) .
$$

The numbers $m_{i}\left(m_{i} \geq 2\right)$ are called the proper periods, the brackets $\left(n_{i 1}, \ldots, n_{i s}\right)\left(n_{i j} \geq 2\right)$ are the period cycles and $g \geq 0$ the orbit genus. It turns out that a signature determines an algebraic presentation of the group [13], [23]. Namely, the group $\Lambda$ with signature (2.1) has the presentation with the following generators:

$$
\begin{array}{lll}
\text { (i) } x_{i}, & i=1, \ldots, r, \\
\text { (ii) } c_{i, j}, & i=1, \ldots, k, \quad j=0, \ldots, s_{i}, \\
\text { (iii) } e_{i}, & i=1, \ldots, k, \\
\text { (iv) } a_{i}, b_{i}, & i=1, \ldots, g \quad \text { if the sign is " }+", \\
& d_{i}, & i=1, \ldots, g \quad \text { if the sign is " }-",
\end{array}
$$


subject to the relations:

(i) $x_{i}^{m_{i}}=1, \quad i=1, \ldots, r$,

(ii) $c_{i, s_{i}}=e_{i}^{-1} c_{i, 0} e_{i}, \quad i=1, \ldots, k$,

(iii) $c_{i, j-1}^{2}=c_{i, j}^{2}=\left(c_{i, j-1} c_{i, j}\right)^{n_{i j}}=1, \quad i=1, \ldots, k, j=1, \ldots, s_{i}$,

(iv) $x_{1} \ldots x_{r} e_{1} \ldots e_{k}\left[a_{1}, b_{1}\right] \ldots\left[a_{g}, b_{g}\right]=1$ if the sign is " + ", $x_{1} \ldots x_{r} e_{1} \ldots e_{k} d_{1}^{2} \ldots d_{g}^{2}=1 \quad$ if the sign is " - ".

In the whole paper these generators will be called the canonical generators of $\Lambda$. Every NEC-group has a fundamental region whose hyperbolic area depends only on the signature of the group and for an NEC-group $\Lambda$ with signature (2.1) it is given by

$$
\begin{aligned}
\mu(\tau) & =\mu(\Lambda) \\
& =2 \pi\left(\alpha g+k-2+\sum_{i=1}^{r}\left(1-1 / m_{i}\right)+\sum_{i=1}^{k} \sum_{j=1}^{s_{i}}\left(1-1 / n_{i j}\right) / 2\right),
\end{aligned}
$$

where $\alpha=1$ if the sign is "-" and $\alpha=2$ in the other case.

It is known that a group $\Lambda$ with presentation (2.2) can be realized as an NEC-group with signature (2.1) if and only if the right hand side of (2.3) is a positive rational.

If $\Gamma$ is a subgroup of finite index in an NEC-group $\Lambda$, then it is also an NEC-group and the following Hurwitz-Riemann formula holds:

$$
[\Lambda: \Gamma]=\mu(\Gamma) / \mu(\Lambda) \text {. }
$$

An NEC-group with signature

$$
(g ; \pm ;[-],\{(-), \ldots ., .,(-)\})
$$

will be called a surface group of genus $g$ with $k$ boundary components, bordered if $k>0$, unbordered if $k=0$, and orientable or nonorientable according as the sign is "+" or "-". If $\Gamma$ is bordered or nonorientable then the number $\mathbf{q}=\alpha g+k-1$, where $\alpha=1$ if the sign is "-" and $\alpha=2$ if the sign is "+", is called the algebraic genus of $\Gamma$. If $k=0$ and the sign is "+" then the algebraic genus $\mathbf{q}$ is, by definition, equal to the orbit genus $g$.

If $\Lambda$ is a proper NEC-group with signature (2.1) then, by [21], $\Lambda^{+}$has signature

$$
\left(g ; \pm ;\left[m_{1}, m_{1}, \ldots, m_{r}, m_{r} n_{11}, \ldots, n_{k s_{k}}\right] ;\{-\}\right) .
$$

It is known [19] that a compact Klein surface of genus $\mathbf{q} \geq 2$ can be represented as $\mathbb{C}^{+} / \Gamma$, where $\Gamma$ is a surface group of algebraic genus q. Moreover, given a bordered Klein surface so represented, a finite group $G$ is a group of its automorphisms if and only if $G=\Lambda / \Gamma$ for some NEC-group $\Lambda$ [15]. 
Observe that a surface group $\Gamma$ of algebraic genus $\mathbf{q}$ has area $2 \pi(\mathbf{q}-1)$. Therefore from the Hurwitz-Riemann formula it follows that the problem of finding an upper bound for the order of a group of automorphisms (lying in a certain family $\mathcal{F}$ of finite groups) of a Klein surface of algebraic genus $\mathbf{q} \geq 2$ is equivalent to the problem of finding a lower bound for the area of NEC-groups $\Lambda$ for which there exists a surface group $\Gamma$ which is a normal subgroup of $\Lambda$ (with $\Lambda / \Gamma \in \mathcal{F}$ ).

We shall need the following result due to Singerman [20].

THEOREM 2.1. A necessary and sufficient condition for a finite group $G$ to act as a group of automorphisms on a nonorientable Riemann surface $X$ of algebraic genus $\mathbf{q}(\mathbf{q} \geq 2)$ is that there exists a proper $N E C$-group $\Lambda$ and a homomorphism $\theta$ from $\Lambda$ onto $G$ with $\theta\left(\Lambda^{+}\right)=G$ and with $\Gamma=\operatorname{Ker} \theta$ being an unbordered surface group. The algebraic genus $\mathbf{q}$ of $X$ equals that of $\Gamma$ and can be calculated using the Hurwitz-Riemann formula.

The next result follows easily from Macbeath's result in [13] that the only elements of finite order in an NEC-group $\Lambda$ are those that are conjugate to nontrivial powers of $c_{i j}, c_{i, j-1} c_{i j}, x_{i}$.

THEOREM 2.2. Let $\Lambda$ be an NEC-group and let $\theta$ be a homomorphism from $\Lambda$ onto a finite group $G$. Then $\Gamma=\operatorname{Ker} \theta$ is an unbordered surface group if and only if $\theta$ preserves proper periods and period cycles, i.e.

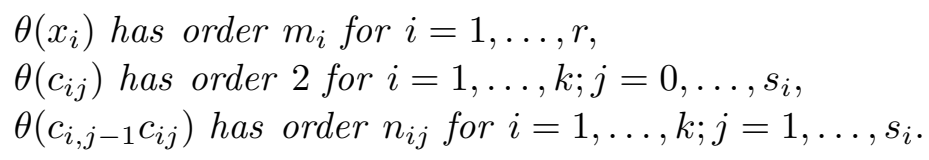

3. On known results concerning nonorientable bordered Klein surfaces with maximal symmetry and with a soluble $M^{*}$-group of automorphisms. As was announced in the introduction there is a connection (see Section 5 for details) between nonorientable Riemann surfaces with a soluble group of automorphisms of greatest possible order and bordered nonorientable Klein surfaces with maximal symmetry and a soluble group of automorphisms.

It was shown by May [14] (see also [18]) that a group of automorphisms of a compact Klein surface $X$ of algebraic genus $\mathbf{q} \geq 2$ has at most $12(\mathbf{q}-1)$ elements and that this bound is attained for infinitely many values of $\mathbf{q}$ [15]. The problem of the classification of topological types (called species) of the corresponding Klein surfaces, which for obvious reasons are said to have maximal symmetry, seems to be enormous, though some parts of it are more approachable. Recently for instance May [17] has classified topologically such surfaces with supersoluble groups of automorphisms. The problem of such a classification of nonorientable surfaces with soluble groups of 
automorphisms was posed in [16]. In the next section we solve it for groups whose derived length does not exceed 4.

By an $M^{*}$-group we mean the automorphism group of a Klein surface with maximal symmetry. It was shown in [10] that a finite group $G$ is an $M^{*}$-group if and only if it can be generated by three elements $a, b, c$ of order 2 such that $a b$ and $b c$ have orders 2 and 3 respectively. The order $s$ of $a c$ is said to be an index of $G$, and the corresponding surface $X$ with maximal symmetry on which $G$ acts has $k=|G| /(2 s)$ boundary components. Moreover, Singerman [22] showed that in that case $X$ is nonorientable if and only if $a b$ and $b c$ generate the whole group $G$.

Summing up, we see that the problem of the topological classification of nonorientable bordered Klein surfaces with maximal symmetry is equivalent to the following purely group-theoretical one:

Given s find all possible orders of groups $G$ that can be generated by three elements $a, b$ and $c$ of order 2 such that $a b, b c$ and ac have orders 2,3 and $s$ respectively and in addition $a b$ and ac generate the whole group $G$.

Let $\Omega$ be a group with the presentation

$$
\left\langle u, v, w \mid u^{2}, v^{2}, w^{2},(u v)^{2},(v w)^{3}\right\rangle
$$

and let $\Omega_{0}$ be its subgroup generated by $u v$ and $v w$. Then $\Omega_{0}$ and $\Omega$ are known to be isomorphic to the modular and extended modular group and so in particular the first is known to be the free product of two cyclic groups of orders 2 and 3 generated by $u v$ and $v w$ respectively. The importance of $\Omega$ in the study of $M^{*}$-groups follows from

LEMMA 3.1. A finite group $G$ of order greater than 6 is an $M^{*}$-group if and only if it is a homomorphic image of $\Omega$.

Proof. The necessity is obvious. So let $G \cong \Omega / K$ be a factor group of order greater than 6 . Then it is easy to see that if some of $u, v, w, u v$, $v w$ belong to $K$ then $|\Omega / K| \leq 6$. So the images $\widetilde{u}, \widetilde{v}$ and $\widetilde{w}$ of $u, v$ and $w$ respectively make $G$ an $M^{*}$-group.

COROLlary 3.2. Let $H$ be a normal subgroup of an $M^{*}$-group $G$ of index greater than 6 . Then $G / H$ is also an $M^{*}$-group. Moreover, if $G$ acts on a nonorientable Klein surface then so does $G / H$.

It was shown by May [14] that there is a dianalytic structure making a real projective plane with three boundary components a Klein surface with maximal symmetry, having $S_{4}$ as the automorphism group. This surface $X_{3}^{-}$ has the important property that every nonorientable Klein surface $X$ with maximal symmetry and with a soluble group of automorphisms is its full cover [10]. So, in particular, if $G$ is the automorphism group of $X$ then there exists a normal subgroup $H$ of $G$ such that $G / H \cong S_{4}$ and $X / H=X_{3}^{-}$. 
In particular, the index of $G$ must be a multiple of 4 since the index of $S_{4}$ corresponding to its action on $X_{3}^{-}$is 4 . Starting with this surface May proved in [15] the following

Proposition 3.3. Given an odd number $m$ there exists a nonorientable Klein surface with maximal symmetry of algebraic genus $\mathbf{q}_{m}=2 m^{3}+1$ whose automorphism group $G_{m}$ is a soluble $M^{*}$-group of derived length 4 .

On the other hand, it is known [16] that there are no other topologically different nonorientable Klein surfaces, with maximal symmetry and with soluble automorphism group, of algebraic genus $\mathbf{q}$ in the range $2 \leq \mathbf{q} \leq 40$. In the next section we shall show that the surfaces from Proposition 3.3 are the only ones with soluble automorphism group of derived length 4 .

Proposition 3.4. Let $G$ be a soluble group of derived length 3 acting as an $M^{*}$-group on a nonorientable bordered Klein surface $X$ with maximal symmetry. Then $G=S_{4}$, it has index 4 , and $X$ is a real projective plane with 3 boundary components.

Pr o of. Let $G=\Omega / K$. Since $G$ has three generators $a, b$ and $c$ of order 2 such that $a b$ and $b c$ have orders 2 and 3 respectively and they generate the whole group $G, G / G^{\prime} \cong \mathbb{Z}_{2}$. By Lemma 1 in [10], $G^{\prime}$ is generated by two elements of order 3 . Thus either $\left|G^{\prime} / G^{\prime \prime}\right|=9$ or $\left|G^{\prime} / G^{\prime \prime}\right|=3$. But the former case is impossible since $G / G^{\prime \prime}$ would then have order 18 , and so it would be an $M^{*}$-group by Corollary 3.2 , a contradiction since on the other hand 12 divides the order of any $M^{*}$-group. So $\left|G / G^{\prime \prime}\right|=6$ and thus by Lemma 4.2 .11 in [4], $G / G^{(3)}=G$ has order 12,24 or 48 . Therefore the result follows from Theorem 8 in [16].

4. Classification of bordered nonorientable Klein surfaces with maximal symmetry and with soluble automorphism group of derived length 4

Proposition 4.1. Let $G$ be a soluble group of derived length 4 acting as the automorphism group on a nonorientable bordered Klein surface with maximal symmetry. Then $G$ has index $4 m$ and order $24 m^{3}$ for some odd integer $m$.

Proof. Let $G$ be a soluble $M^{*}$-group of derived length $n(n \geq 4)$ acting on a nonorientable bordered Klein surface with maximal symmetry and let $H=G^{(3)}$ be the third derived group of $G$. By Proposition 3.4, $G / H=S_{4}$ and it still acts as an $M^{*}$-group on a nonorientable Klein surface with maximal symmetry. Let us represent $G$ and $S_{4}$ as quotients $\Omega / K$ and $\Omega / L$ respectively. We shall see how the groups $K$ and $L$ look like. Clearly $H=L / K$ has derived length $n-3$. 
We know that $u w$ induces in $\Omega / L$ an element of order 4 . As $\Omega / L$ is isomorphic to $S_{4}$, uvw represents in $\Omega / L$ an element of order 3 . Thus the element $A$ of $\Omega$ represented by $(u v w)^{3}$ belongs to $L$. Denote by $N$ the normal closure in $\Omega$ of $A$. Then by [9], $\Omega / N \cong S_{4}$ and so $L=N$. Now let $B=u A u, C=v w u A u w v$ and denote by $M$ the subgroup of $\Omega$ generated by $A, B$ and $C$. Then it is easy to check, using the defining relations for $\Omega$, that $\Omega$ acts on $M$ as follows:

$$
\begin{gathered}
u A u=B, \quad u B u=A, \quad u C u=(A C B)^{-1}, \\
v A v=B^{-1}, \quad v B v=A^{-1}, \quad v C v=C^{-1}, \\
w A w=A^{-1}, \quad w B w=C^{-1}, \quad w C w=B^{-1} .
\end{gathered}
$$

Thus $M$ is a normal subgroup of $\Omega$ and so $M=N$. Therefore $L=\langle A, B, C\rangle$.

We claim that $L$ is free. Indeed, consider the automorphism $\phi$ of $\Omega$ induced by the assignment

$$
\phi(u)=u v, \quad \phi(v)=v, \quad \phi(w)=w .
$$

Then $\phi(A)=A^{\prime}=(u w)^{3}, \phi(B)=B^{\prime}=v(w u)^{3} v, \phi(C)=C^{\prime}=w v(u w)^{3} v w$ and so $L=\langle A, B, C\rangle$ and $L^{\prime}=\left\langle A^{\prime}, B^{\prime}, C^{\prime}\right\rangle$ are isomorphic. Moreover, $\Omega / L^{\prime} \cong \Omega / L \cong S_{4}$. Observe that $L^{\prime}$ is a subgroup of $\Omega_{0}$ which is the free product of two cyclic groups of orders 2 and 3 generated by $u v$ and $v w$ respectively. Clearly none of the conjugates of $u v$ and $v w$ belongs to $L^{\prime}$ since otherwise $\Omega / L^{\prime}$ would be of order $\leq 6$. Therefore by the Kurosh subgroup theorem $L^{\prime}$ is free and so is $L$. It turns out that $A, B$ and $C$ freely generate $L$, i.e. $L$ has rank 3 . Indeed, looking at the proof of Proposition 3.3 given in [15] we see that the third derived group $G_{m}^{(3)}$ of $G_{m}$ is isomorphic to $\left(\mathbb{Z}_{m}\right)^{3}$ and it is a homomorphic image of $L$.

From (4.1) we see that the images of $A, B$ and $C$ in $H$ have the same order, say $m$.

Now if $G=\Omega / K$ has derived length 4 , then $H=L / K$ is abelian and so we have to study subgroups $K$ of $L$ which are normal in the whole group $\Omega$ and have $L / K$ abelian. We first show that $m$ is odd. Indeed, assume that this is not the case and consider a subgroup $H^{\prime}$ of $H$ of odd order such that $H / H^{\prime}$ is a 2-group. Clearly $H^{\prime}$ is a normal subgroup of $G$ and $G / H^{\prime}$ has order $24 \cdot 2^{\alpha}$, where $\alpha \geq 1$. By Corollary 3.2, $G / H^{\prime}$ acts as an $M^{*}$-group on a nonorientable bordered Klein surface of algebraic genus $\mathbf{q}=2^{\alpha+1}+1$, which contradicts Theorem 6 of [16].

Now we show that $|H|=m^{3}$. It suffices to show that for any prime $p$ and for an integer $\alpha$ such that $m=p^{\alpha} s$, where $p$ and $s$ are relatively prime, $p^{3 \alpha}$ divides $|H|$. Assume that this is not the case for some $p$. Since $H$ is abelian, $H=H_{p} \oplus H_{p^{\prime}}$ where $H_{p}$ is a Sylow $p$-subgroup of $H$. Clearly $H_{p^{\prime}}$ is a normal subgroup of $G$ and $G / H_{p^{\prime}}$ has order $24 \cdot p^{N}$, for some $N \geq 1$. By Corollary 3.2, $G / H_{p^{\prime}}$ acts as an $M^{*}$-group on a nonorientable 
bordered Klein surface with maximal symmetry. For notational simplicity assume that $H_{p^{\prime}}=1$, i.e. assume that $G$ already has order $24 \cdot p^{N}$. Then $H=\mathbb{Z}_{p^{\alpha}} \oplus \mathbb{Z}_{p^{\beta}} \oplus \mathbb{Z}_{p^{\delta}}$, where $0 \leq \alpha \leq \beta \leq \delta$. By our assumption $\alpha \neq \delta$. Let $H_{\delta}=\left\{x \in H: x^{p^{\delta-1}}=1\right\}$. Then $H_{\delta}$ is a normal subgroup of $G$ and either $\widetilde{H}=H / H_{\delta}=\mathbb{Z}_{p}$ or $\widetilde{H}=\mathbb{Z}_{p} \oplus \mathbb{Z}_{p}$. Represent $\widetilde{G}=G / H_{\delta}$ as a quotient $\Omega / K_{1}$. Then $K_{1}<L$ and either $L / K_{1}=\mathbb{Z}_{p} \oplus \mathbb{Z}_{p}$ or $L / K_{1}=\mathbb{Z}_{p}$. Let $\widetilde{A}, \widetilde{B}$ and $\widetilde{C}$ denote the images of $A, B$ and $C$ respectively in $L / K_{1}$. If one of $A, B, C$ belongs to $K_{1}$ then, by (4.2), so do the other ones. So $\widetilde{A} \neq 1, \widetilde{B} \neq 1$ and $\widetilde{C} \neq 1$. There are integers $\alpha, \beta$ and $\delta$ in the range $0 \leq \alpha, \beta, \delta<p$ not all equal to 0 such that $(\widetilde{A})^{\alpha}(\widetilde{B})^{\beta}(\widetilde{C})^{\delta}=1$. Clearly either $\alpha \neq 0$ or $\beta \neq 0$ since otherwise $\widetilde{C}=1$ as $p$ is prime. Since $A^{-\beta} C^{-\delta} B^{-\alpha}=B^{\alpha} v\left(A^{\alpha} B^{\beta} C^{\delta}\right) v B^{-\alpha} \in K_{1}$ we can assume, without loss of generality, that $\alpha \neq 0$. Then since $p$ is an odd prime, $\widetilde{A}=(\widetilde{B})^{b}(\widetilde{C})^{c}$ for some integers $b$ and $c$ with $0 \leq b, c<p$.

Now $A C^{-b} B^{-c}=w\left(A^{-1} \bar{B}^{b} C^{c}\right) w \in K_{1}$ and thus $(\widetilde{B})^{b-c}=(\widetilde{C})^{b-c}$. So either $b=c$ or $\widetilde{B}=\widetilde{C}$. We claim that the first case implies the second and that the latter is impossible. Indeed, if $b=c$ then

$$
1=(\widetilde{A})^{-(b+1)}(\widetilde{A})^{b}(\widetilde{B})^{b}(\widetilde{C})^{b}=(\widetilde{A})^{-(b+1)}(\widetilde{A} \widetilde{C} \widetilde{B})^{b}
$$

and thus $B^{-(b+1)} C^{-b}=u\left(A^{-(b+1)}(A C B)^{b}\right) u \in K_{1}$. So since $B^{-(b+1)} C B^{b}$ is equal to $\left(B^{-(b+1)} C^{-b}\right) w\left(B^{-(b+1)} C^{-b}\right) w$ and the latter belongs to $K_{1}$ we obtain $\widetilde{B}=\widetilde{C}$ as desired.

Now it is easy to check that

$$
B^{4}=\left(B C^{-1}\right)\left(v\left(C^{-1} B\right) v\right)\left(A\left(B C^{-1}\right) A^{-1}\right)\left((u v)\left(C B^{-1}\right)(u v)^{-1}\right) .
$$

So if $\widetilde{B}=\widetilde{C}$ then the right hand side belongs to $K_{1}$. Hence $B^{4} \in K_{1}$ and since $p$ is odd also $B \in K_{1}$, a contradiction. Thus $|H|=m^{3}$.

Finally, notice that $C B=(w u)^{4}$ and $\widetilde{C} \widetilde{B}$ has order $m$. So $G$ has index $4 \mathrm{~m}$. This completes the proof of our proposition.

Combining Propositions 3.3 and 4.1 we obtain

THEOREM 4.2. There exists a nonorientable bordered Klein surface with maximal symmetry of algebraic genus $\mathbf{q}$ with $k$ boundary components and with soluble $M^{*}$-group of automorphisms of derived length 4 if and only if $\mathbf{q}=2 m^{3}+1$, and $k=3 m^{2}$ for some odd integer $m$.

\section{Nonorientable Riemann surfaces with a soluble group of} automorphisms of maximal possible order

THEOREM 5.1. Let $G$ be a soluble group acting as a group of automorphisms on a nonorientable Riemann surface $X$ of algebraic genus $\mathbf{q} \geq 2$. Then $|G| \leq 24(\mathbf{q}-1)$. 
Proof. By Theorems 2.1 and 2.2 there exists a proper NEC-group $\Lambda$ and an epimorphism $\theta: \Lambda \rightarrow G$ preserving proper periods and period cycles such that $\theta\left(\Lambda^{+}\right)=\Gamma$. Let $\Gamma=\operatorname{Ker} \theta$. By $(2.3), \mu(\Gamma)=2 \pi(\mathbf{q}-1)$ and by the Hurwitz-Riemann formula $|G|=\mu(\Gamma) / \mu(\Lambda)=2 \pi(\mathbf{q}-1) / \mu(\Lambda)$. Therefore the proof will be complete when we show that $\mu(\Lambda) \geq \pi / 12$.

Let $\tau$ be the signature of $\Lambda$ and assume that $\tau$ has the general form (2.1). Since $\Lambda$ is a proper NEC-group, either $k \neq 0$ or $g \neq 0$. Moreover, we can assume that $\alpha g+k-1=0$ since otherwise either $\mu(\tau)=0$ or $\mu(\tau) \geq \pi / 2$. So we have either $g=1, \alpha=1, k=0$, or $g=0, k=1$. In the first case $r \geq 2$ since otherwise $\mu(\tau)<0$. However, if $r \geq 3$ then $\mu(\tau) \geq \pi / 2$ whilst if $r=2$ then $\mu(\tau)=0$ for $m_{1}=m_{2}=2$ and $\mu(\tau) \geq \pi / 3$ otherwise.

So we can assume that $g=0$ and $k=1$. Now if $r \geq 3$ then $\mu(\tau) \geq \pi$. If $r=2$ and either $m_{1} \neq 2$ or $m_{2} \neq 2$ then $\mu(\tau) \geq \pi / 3$ whilst if $m_{1}=m_{2}=2$ then the period cycle is nonempty and in this case $\mu(\tau) \geq \pi / 2$. So let $r=1$. Then if $s \geq 3, \mu(\tau) \geq \pi / 2$. If $s=2$ then $\mu(\tau)=0$ if $m_{1}=n_{1}=n_{2}=2$ and $\mu(\tau) \geq \pi / 6$ otherwise. Thus let $r=s=1$. Then $\mu(\tau)<0$ for $m_{1}=2$, $\mu(\tau) \geq \pi / 3$ for $m_{1} \geq 4$ and $\mu(\tau)=\pi / 12$ for $m=3, n=4$, i.e. for $\tau=(0 ;+;[3] ;\{(4)\})$.

Therefore let $r=0$. Then $\mu(\tau) \geq \pi / 2$ if $s \geq 5$. If $s=4$ and $n_{1}=n_{2}=$ $n_{3}=n_{4}=2$ then $\mu(\tau)=0$, whilst $\mu(\tau) \geq \pi / 6$ otherwise. If $s \leq 2$ then $\mu(\tau)<0$. Thus we can assume that $s=3$, i.e.

$$
\tau=\left(0 ;+;[-] ;\left\{\left(n_{1}, n_{2}, n_{3}\right)\right\}\right) .
$$

Clearly the presentation of an NEC-group with the above signature does not depend on the ordering of $n_{1}, n_{2}, n_{3}$ and the reader can easily check that, up to this ordering, the only NEC signatures $\tau$ of the above form for which $\mu(\tau)<\pi / 12$ are $\tau_{n}=(0 ;+;[-] ;\{(2,3, n)\})$ where $n=7,8,9,10,11$, and $\mu\left(\tau_{12}\right)=\pi / 12$.

An NEC-group $\Lambda_{n}$ with signature $\tau_{n}$ is generated by three reflections $c_{0}$, $c_{1}, c_{2}$ subject to the relations $\left(c_{0} c_{1}\right)^{2}=\left(c_{1} c_{2}\right)^{3}=\left(c_{0} c_{2}\right)^{n}=1$ and $\Lambda^{+}$is generated by $c_{0} c_{1}, c_{1} c_{2}$. By Theorem 2.2, $a=\theta\left(c_{0}\right), b=\theta\left(c_{1}\right)$ and $c=\theta\left(c_{2}\right)$ are of order 2 , and $a b, b c$ and $a c$ have orders 2,3 and $n$ respectively. Therefore $G$, the group generated by $a, b, c$, is an $M^{*}$-group with index $n$. Now since $\Gamma$ is nonorientable, $a b$ and $b c$ generate $G$. Thus $G$ acts as an $M^{*}$-group with index $n$ on a nonorientable Klein surface with maximal symmetry. From the remark preceding Proposition 3.3 we know that 4 divides $n$. So $n \neq 7,9,10,11$ and it remains to rule out the case $n=8$.

Notice first that in that case $G^{(3)} \neq 1$, since otherwise $G=S_{4}$, by Proposition 3.4, and so $a c$ would be an element of order 4 . Let $\widetilde{G}=G / G^{(4)}$. Then $|\widetilde{G}|>24$. If some of $a, b, c, a b, b c, a c,(a c)^{2},(a c)^{4}$ belongs to $G^{(4)}$ then $|\widetilde{G}| \leq 24$. So $\widetilde{G}$ is a soluble group of derived length 4 acting as an $M^{*}$-group with index 8 on a nonorientable bordered Klein surface with 
maximal symmetry, a contradiction with Proposition 4.1 that turns out to be crucial here. This finishes the proof.

R e mark. We have observed in the proof of the above theorem that if $G$ is a group of order $24(\mathbf{q}-1)$ that acts as a group of automorphisms on a nonorientable Riemann surface of algebraic genus $\mathbf{q}$ then $G=\Lambda / \Gamma$, where $\Gamma$ is an unbordered nonorientable surface group with area $2 \pi(\mathbf{q}-1)$ and $\Lambda$ is an NEC-group with signature

$$
\tau=(0 ;+;[3] ;\{(4)\}) \quad \text { or } \quad(0 ;+;[-] ;\{(2,3,12)\}) .
$$

Now we rule out the first case.

Proposition 5.2. There is no factor group $G=\Lambda / \Gamma$ such that $G$ is soluble, $\Gamma$ is a nonorientable unbordered surface group, and $\Lambda$ is an $N E C$ group with signature $\tau=(0 ;+;[3] ;\{(4)\})$.

In the proof of 5.2 we shall need the following technical

LEMMA 5.3 ([5]). A finite nilpotent group $G$ cannot be generated by three elements $a, b$ and $c$ of order 2 such that $a b$ and $b c$ generate the whole $G$ and $a b, b c$ and $a c$ have orders $2, k$ and $l$ respectively, where $k$ and $l$ are greater than 2 .

Proof of 5.2. Assume on the contrary that such a factor exists and denote by $\theta$ the canonical projection $A G$. Let $G$ have the smallest possible order. By Theorem 2.1, $G=\theta\left(\Lambda^{+}\right) \cong \Lambda^{+} / \Gamma^{+}$and by $(2.6), \Lambda^{+}$has signature $(0 ;+;[3,3,4] ;\{-\})$. So $G / G^{\prime} \cong \mathbb{Z}_{3}$. Let $G^{\prime}=\Lambda_{1} / \Gamma$ for some NECgroup $\Lambda_{1}$. Then $\Lambda / \Lambda_{1} \cong \mathbb{Z}_{3}$ and so by Theorem 2.5 in [3], $\Lambda_{1}$ has signature $(0 ;+;[-] ;\{(4,4,4)\})$. By Theorem 2.1 the images of $c_{0} c_{1}$ and $c_{1} c_{2}$ generate $G^{\prime}$. On the other hand, $G^{\prime}$ is generated by elements of order 2. So, in particular, either $G^{\prime} / G^{\prime \prime} \cong \mathbb{Z}_{2}$ or $G^{\prime} / G^{\prime \prime} \cong \mathbb{Z}_{2} \oplus \mathbb{Z}_{2}$. In the former case $G / G^{\prime \prime} \cong D_{3}$. By Theorem 2.1, $G$ is generated by $\theta(x)$ and $\theta\left(c_{0} c_{1}\right)$. So $\theta\left(c_{0}\right) \theta\left(c_{1}\right) \notin G^{\prime \prime}$ and thus $\theta\left(c_{0} c_{1}\right)$ induces in $G / G^{\prime \prime}$ an element of order 3 since none of $\theta\left(c_{0}\right)$, $\theta\left(c_{1}\right)$ belongs to $G^{\prime \prime}$ as $c_{0}$ and $c_{1}$ are conjugate in $\Lambda$. On the other hand, $c_{0} c_{1}$ is an element of order 4 and hence the order of $\theta\left(c_{0} c_{1}\right)$ divides 4 , a contradiction. Therefore $G / G^{\prime \prime} \cong \mathbb{Z}_{2} \oplus \mathbb{Z}_{2}$. Inspecting the derived series of the three nonabelian groups of order 12 we see that $G / G^{\prime \prime} \cong A_{4}$.

We claim that $G^{\prime \prime}$ is abelian. Indeed, assume that $G^{(3)} \neq 1$. Then $|G|>$ $\left|G / G^{(3)}\right|$. If one of $\theta\left(c_{0}\right), \theta\left(c_{1}\right)$ belongs to $G^{(3)}$ then, since $c_{0}$ and $c_{1}$ are conjugate, so does the other and hence $G / G^{(3)} \cong \mathbb{Z}_{3}$. Thus $\theta\left(c_{0}\right), \theta\left(c_{1}\right) \notin G^{(3)}$. We shall see that $\theta\left(c_{0} c_{1}\right)^{2} \notin G^{(3)}$. Indeed, if this were not so then the images $a, b$ and $c$ of $\theta(x), \theta\left(c_{0}\right)$ and $\theta\left(c_{1}\right)$ in $G / G^{(3)}$ would have orders 3,2 and 2 respectively, $b c$ would have order 2 and in addition $a$ and $b c$ would generate $G / G^{(3)}$. Using the defining relations for $\Lambda$ we see that $\left(c_{0} c_{1} x\right)^{3}=1$. So $a b c$ has order 3 . But the group generated by two elements of orders 2 and 3 
whose product has order 3 is obviously isomorphic to $A_{4}$, a contradiction. Thus $b c$ has order 4 and so by Theorems 2.1 and $2.2, G / G^{(3)}=\Lambda / \Gamma_{1}$ for some nonorientable surface group, which contradicts the minimality of $G$. So $G^{\prime \prime}$ is abelian.

Now we show that $G^{\prime}$ is a 2-group. In fact, $G^{\prime}=\Lambda_{1} / \Gamma \cong \Lambda_{1}^{+} / \Gamma^{+}$. By $(2.6), \Lambda_{1}^{+}$has signature $(0 ;+;[4,4,4] ;\{-\})$. We already know that $G^{\prime} / G^{\prime \prime} \cong \mathbb{Z}_{2} \oplus \mathbb{Z}_{2}$. Let $G^{\prime \prime}=\Lambda_{2} / \Gamma^{+}$for some NEC-group $\Lambda_{2}$ contained in $\Lambda_{1}^{+}$. Then $\Lambda_{1}^{+} / \Lambda_{2} \cong \mathbb{Z}_{2} \oplus \mathbb{Z}_{2}$ and so, by Corollary 2.3 in [2] and by the Hurwitz-Riemann formula, $\Lambda_{2}$ has signature $(0 ;+;[2,2,2,2,2,2] ;\{-\})$. So, in particular, $G^{\prime \prime}$ is generated by elements of order 2 and since it is abelian we deduce that $G^{\prime}$ is a 2 -group.

Now from $G^{\prime}=\Lambda_{1} / \Gamma$ and from Theorems 2.1 and 2.2 we see that $G^{\prime}$ has three elements $A, B$ and $C$ of order $2, A B, B C$ and $A C$ have order 4 and any two of $A B, B C, A C$ generate $G^{\prime}$. We claim that this is impossible.

Indeed, assume that $H$ is a 2-group so generated of smallest possible order. It is easy to see that there are no such groups of orders 8 and 16 . Thus $|H| \geq 32$. Let $H_{0}$ be a normal subgroup of $H$ of order 2. By the minimality of $H$ at least one of $A, B, C,(A B)^{2},(B C)^{2},(A C)^{2}$ belongs to $H_{0}$. But if one of $A, B, C$ belongs to $H_{0}$ then clearly $\left|H / H_{0}\right| \leq 8$, a contradiction. Now if $(A B)^{2} \in H_{0}$ then, since $A B$ and $B C$ generate $H$, one of $(B C)^{2},(A C)^{2}$ belongs to $H_{0}$, by Lemma 5.3 , and so $\left|H / H_{0}\right| \leq 8$, a contradiction again. This completes the proof.

Combining Theorems 2.1, 2.2, Proposition 5.2 and the remark preceding it we obtain at once the following

COROLlARY 5.4. Let $G$ be a soluble group of order $24(\mathbf{q}-1)$ acting as a group of automorphisms on a nonorientable Riemann surface of algebraic genus $\mathbf{q}$. Then $G$ is an $M^{*}$-group with index 12 acting on a nonorientable bordered Klein surface with maximal symmetry of algebraic genus $\mathbf{q}^{\prime}=2 \mathbf{q}-$ 1 , having $k=\mathbf{q}-1$ boundary components.

We know from Proposition 2.4 that there is no soluble group of derived length 3 acting as an $M^{*}$-group with index 12 on a nonorientable bordered Klein surface with maximal symmetry. Moreover, by Theorem 4.2, such a group $G$ of derived length 4 exists if and only if its order is assumed to be $24 \cdot 3^{3}$. So we have the following

THEOREM 5.5. (1) A soluble group of derived length $d \leq 3$ acting as a group of automorphisms on a nonorientable Riemann surface of algebraic genus $\mathbf{q} \geq 2$ has strictly fewer than $24(\mathbf{q}-1)$ elements.

(2) For $d=4$ the bound $24(\mathbf{q}-1)$ is attained if and only if $\mathbf{q}=3^{3}+1$.

Using a construction of Singerman (see Section 5 of [20]) one can easily prove the following 
THEOREM 5.6. For any odd integer $m$ and for $\mathbf{q}=27 m^{28}+1$ there exists a nonorientable Riemann surface of algebraic genus $\mathbf{q}$ admitting a soluble group of automorphisms of derived length 5 and of order $24(\mathbf{q}-1)$.

Finally, observe that starting with the above result and iterating the construction of Singerman, one can show that the bound $24(\mathbf{q}-1)$ is attained for soluble groups of any derived length $d \geq 5$ for infinitely many values of $\mathbf{q}$.

We finish the paper with the following problems concerning Theorem 5.5 (see [8] and [11] for results concerning orientable Riemann surfaces).

Problems. (1) Given $d \leq 3$, find a bound for the order of a soluble group of derived length $d$ acting on a nonorientable Riemann surface of algebraic genus $\mathbf{q} \geq 2$.

(2) Find a bound for the order of a soluble group of derived length 4 acting on a nonorientable Riemann surface of algebraic genus $\mathbf{q} \geq 2, \mathbf{q} \neq$ $3^{3}+1$.

Acknowledgment. I would like to thank the referee for some helpful suggestions.

\section{References}

[1] N. L. Alling and N. Greenleaf, Foundations of the Theory of Klein Surfaces, Lecture Notes in Math. 219, Springer, 1971.

[2] E. Bujalance, Proper periods of normal NEC subgroups with even index, Rev. Math. Hisp.-Amer. 41 (4) (1981), 121-127.

[3] -, Normal subgroups of NEC groups, Math. Z. 178 (1981), 331-341.

[4] E. Bujalance, J. J. Etayo, J. M. Gamboa and G. Gromadzki, Automorphism Groups of Compact Bordered Klein Surfaces, Lecture Notes in Math. 1439, Springer, 1990.

[5] E. Bujalance and G. Gromadzki, On nilpotent groups of automorphisms of compact Klein surfaces, Proc. Amer. Math. Soc. 108 (3) (1990), 749-759.

[6] B. P. Chetiya, Groups of automorphisms of compact Riemann surfaces, Ph.D. thesis, Birmingham University, 1981.

[7] -, On genuses of compact Riemann surfaces admitting solvable automorphism groups, Indian J. Pure Appl. Math. 12 (1981), 1312-1318.

[8] B. P. Chetiya and K. Patra, On metabelian groups of automorphisms of compact Riemann surfaces, J. London Math. Soc. 33 (1986), 467-472.

[9] H. S. M. Coxeter and W. O. J. Moser, Generators and Relations for Discrete Groups, 3rd ed., Ergeb. Math. Grenzgeb. 14, Springer, Berlin 1972.

[10] N. Greenleaf and C. L. May, Bordered Klein surfaces with maximal symmetry, Trans. Amer. Math. Soc. 274 (1982), 265-283.

[11] G. Gromadzki, On soluble groups of automorphisms of Riemann surfaces, Canad. Math. Bull. 34 (1) (1991), 67-73.

[12] A. Hurwitz, Ueber algebraische Gebilde mit eindeutigen Transformationen in sich, Math. Ann. 41 (1893), 403-442. 
[13] A. M. Macbeath, The classification of non-euclidean plane crystallographic groups, Canad. J. Math. 19 (1967), 1192-1205.

[14] C. L. May, Automorphisms of compact Klein surfaces with boundary, Pacific J. Math. 59 (1975). 199-210.

[15] - Large automorphism groups of compact Klein surfaces with boundary I, Glasgow Math. J. 18 (1977), 1-10.

[16] - The species of Klein surfaces with maximal symmetry of low genus, Pacific J. Math. 111 (2) (1984), 371-394.

[17] —, Supersolvable $M^{*}$-groups, Glasgow Math. J. 30 (1988), 31-40.

[18] K. Oikawa, Note on conformal mappings of a Riemann surface onto itself, Kodai Math. Sem. Rep. 8 (1956), 23-30.

[19] R. Preston, Projective structures and fundamental domains on compact Klein surfaces, Ph.D. thesis, Univ. of Texas, 1975.

[20] D. Singerman, Automorphisms of compact non-orientable Riemann surfaces, Glasgow Math. J. 12 (1971), 50-59.

[21] - On the structure of non-Euclidean crystallographic groups, Proc. Cambridge Philos. Soc. 76 (1974), 233-240.

[22] - Orientable and non-orientable Klein surfaces with maximal symmetry, Glasgow Math. J. 26 (1985), 31-34.

[23] M. C. Wilkie, On non-euclidean crystallographic groups, Math. Z. 91 (1966), 87102.

INSTITUTE OF MATHEMATICS

PEDAGOGICAL UNIVERSITY (WSP)

CHODKIEWICZA 30

85-064 BYDGOSZCZ, POLAND

Received 26 November 1990;

in revised form 11 February 1992 